\title{
Principios normativos en la política social
}

\author{
Maingon, Thaís*
}

\section{Resumen}

Este trabajo intenta exponer, desde una perspectiva exploratoria, un conjunto de consideraciones sobre algunas formulaciones teóricas que sinan como un marco de reflexión amplio sobre los principios normativos considerados como los primordiales y que no deben faltar en cualquier proceso de construcción y diseño de una la política social. La base argumentativa analítica metodológica privilegiada fue un grupo de suposiciones y aportes que sobre este punto ha sido expuesto por algunos autores considerados como fundamentales en cualquier proceso de reflexión que se haga sobre este tema. En medio de la multiplicidad de aproximaciones a los tipos de principios que intervienen en la formulación de políticas sociales, seleccionamos tres de ellos que pueden considerarse como los más relevantes en estos procesos. Se trata de los principios de eficacia, eficiencia y equidad. Lo que aqui se presenta, de ninguna manera pretende enunciar teoría alguna, sino más bien señalar una serie de suposiciones teórico-metodológicas sobre estos principios y su relación con el proceso de formulación de la política social. De estas líneas se desprende, entre otras cuestiones, que en un proceso de toma de decisiones que tenga que ver directamente con la formulación de una deterninada política social, los actores responsables de su diseño, pueden privilegiar uno de estos criterios por sobre los otros.

Palabras clave: Política social, principios normativos, toma de decisiones, eficiencia, eficacia, equidad.

\section{Normative Principles in Social Politics}

\section{Abstract}

This paper attempts to explain from an exploratory perspective a set of considerations as to certain theoretical precepts that serve as an extensive reflective framework as to the normative principles considered as primary and which must be taken into consideration in any process of social policy design. The argumentative, analytical and methodological basis considered was a set of suppositions and contributions on this topic that have been advanced by certain authors considered

Recibido: 01-01-08, Aceptado: 01-05-02

Investigadora del CENDES (UCV-Venezuela) Area de Desarrollo Socio-Politico, E-mail: tmaigon reacciun. ve. La autora agradece las observaciones a los Profesores Carlos Aponte y Jesús Hernández. 
as fundamental to any process of reflection on this theme. Amidst the multiplicity of approximations made towards the types of principles that intervene in the formulation of social policies, three were chosen which could be considered as relevant to these processes. They are the principles of efficacy (utility), efficiency and equity. What is presented by no means pretends to announce a theory per se, but only to point out a series of theoretical-methodological suppositions on these principles and on their relation to the process of social policy formulation. In this paper we understand, among other things, that in a decision-making process that has to do directly with the formulation of a specific social policy, the agents responsible for the design should consider above all one of these three criteria.

Key words: Social policy, normative principles, decision making, efficiency, efficacy, equity.

\section{Introducción}

En este trabajo se hará una reflexión sobre la incidencia de los principios normativos en las decisiones de política social $y$, en especial, sobre la influencia de esos principios en la justificación de los fines y medios que se seleccionan para el desarrollo de esa política. Los principios normativos subyacen como criterios sujetos a debate que orientan las decisiones y le imprimen una dirección específica al proceso de formulación e instrumentación de una política social. Aunque el concepto de principio normativo ha sido considerado como un concepto central para el análisis del proceso de formulación y evaluación de una política social, no existe un uso unitario respecto a su significado y definición.

En medio de esa diversidad, desde el punto de vista conceptual, puede ser especialmente útil considerar la defini- ción de principios de Elster (1994: 77) que se ajusta particularmente a las orientaciones analíticas que nos proponemos desarrollar. Siguiendo a este autor, entenderemos por principios todos aquellos criterios que generan procedimientos para guiar la distribución y asignación del beneficio escaso (como consecuencia de las decisiones que se dan en el proceso de selección de los medios y la formulación de los objetivos de una política social). Los procedimientos son la versión operacional de un principio dentro de una politica. El conjunto de procedimientos conforma el modelo de distribución y/o redistribución implicito o explicito de la política a instrumentarse. Argumenta que "como un principio siempre puede implementarse mediante varios procedimientos diferentes, no determina completamente por si sólo quiénes han de ser los receptores"1.

A los elementos que guian un esquema general de distribución, el autor los denomina el conjunto total de los procedimientos utilizados para instrumentar los principios que usualmente son mixtos y a veces contradictorios entre sí. Finalmente, Elster señala que las asignaciones no pueden reducirse a un solo principio, sino que más bien es un proceso que tiene como base una combinación de criterios y seguidamente anota que no todas las veces los recursos escasos se asignan mediante principios sino que más bien las asignaciones se realizan de un modo discrecional, sin principio alguno que los guie. Intuimos que, en este caso, está haciendo referencia a los procesos de asignación asociados con la corrupción y el clientelismo, entre otros. 
Pueden destacarse algunas características básicas atribuibles a los principios normativos:

- Los principios se refieren al deber ser, a lo que sería lo ideal, por lo que no pretenden describir acciones y conductas de determinados actores. Cuando calificamos los principios como normativos estamos haciendo referencia a enunciados con naturaleza prescriptiva, enunciados que denotan el deber ser de una política.

- Los principios que estamos calificando como 'normativos' son aquellos que tienen un efecto en las decisiones de los actores responsables de diseñar políticas.

- Los principios orientadores de una politica no son estáticos y están definidos por el movimiento sociopolítico de la relación Estado/sociedad.

- Los principios sirven para evaluar las prácticas en función del grado en que la política se ajusta o no a los principios asumidos como válidos.

En este aparte se desarrollará un marco de reflexión explicativo sobre los principios normativos presentes en la política social, considerando algunos de los principales aportes de distintos autores a la reflexión sobre los mismos. En medio de la multiplicidad de aproximaciones a los tipos de principios que intervienen en la formulación de políticas sociales, seleccionaremos tres de ellos que pueden considerarse como los más relevantes para los procesos de elaboración, análisis y evaluación de una política social. Se trata de los principios de eficacia, eficiencia y equidad.

Esos principios se combinan de diversas maneras en las políticas sociales $y$, en ocasiones, puede atribuírsele un predominio a alguno de los mismos en la orientación de una política. Conocer estos principios es significativo para entender la justificación del proceso general de toma de decisiones, así como también para tener la posibilidad de controlar los resultados de la política social y discutir sobre sus fines ${ }^{2}$.

Es importante resaltar que en la escogencia de los principios básicos que se privilegiarán en el análisis podría suponerse que se ha incurrido en una omisión particularmente destacada: la del principio de racionalidad. Sin embargo, concebimos a la racionalidad más que como un principio, como una condición presente en todo proceso de decisión. Como plantea Anderson (1979) la racionalidad está "autodefinida" ya que el proceso de decisión política es un proceso racional ${ }^{3}$.

Sobre este interesante tema véase Barragán 1992.

3 Es oportuno, en esta dimensión introductoria de la reflexión sobre los principios, registrar otros planteamientos de Anderson (1979) quien, siguiendo una argumentación distinta a Elster y en el marco de la teoría de la evaluación política, dirige su atención hacia la selección de los criterios presentes en el proceso de decisión respondiendo a la pregunta de cómo se debería decidir ("how we ougtht to decide") mas que cormo se puede decidir ("how we can decide or how we in fact do decide"). De all que argumente que el proceso de decisión depende, desde su inicio de la definición, de los principios o valores a ser alcanzados por la política a instrumentar. En este sentido, Anderson identifica tres principios clásicos: autoridad, justicia y eficiencia. 
Desde otro espectro del pensamiento político, Dunn (1981) señala que las decisiones políticas involucran lo que llama una base 'multiracional', es decir que el diseño de una política incluiría a mas de un tipo de racionalidad. La primera que menciona es la racionalidad técni$c a$ la cual promueve una selección entre opciones de políticas que sean efectivas para la solución de la situación. La racionalidad económica está en la base de las decisiones políticas que buscan las soluciones eficaces en las intervenciones públicas; este tipo de racionalidad privilegia para la toma de decisiones el total de los costos y de los beneficios que se obtendrán con determinada elección de política. La Racionalidad legal, está presente en aquellas decisiones que privilegian la conformidad legal para establecer reglas y acuerdos. La racionalidad social, es la base de aquellas decisiones que promueven los valores sociales institucionales $y$, por último Dunn, menciona la racionalidad substantiva que es la racionalidad que contempla múltiples formas de racionalidad con el objeto de llegar a la decisión política mas apropiada bajo determinadas circunstancias ${ }^{4}$.

La formulación de una política social no puede obviar el problema de los principios, las opciones y preferencias.
Debe preocuparse por los posibles impactos de la política a instrumentar, por los objetivos y fines y por la forma o los medios de lograr esos objetivos. La interdependencia entre los medios y los fines es ineludible. Frecuentemente, los fines, los procedimientos y también lo referido a los principios generales, cambian debido, principalmente, a la innovación de los medios. Otro factor que influye significativamente en esta relación viene dado por los cambios que se dan en la distribución del poder. Cuando los medios cambian, frecuentemente cambia también la distribución del poder. El cambio de los medios para alcanzar determinados fines puede indicar que hay un cambio en los principios.

Los tres principios mencionados son los principios básicos que están contenidos en el propio concepto de politica social ya discutido. Estos señalan patrones de distribución y caminos para alcanzar el bienestar colectivo. La eficiencia tiene que ver, fundamentalmente, con las cuestiones asociadas a la distribución de los recursos y deberá responder a cómo se distribuyen los recursos para que los individuos satisfagan sus necesidades. La eficacia hace referencia a la capacidad que tiene una determinada política de producir cambios (los esperados, tica ha dado pie a distintos enfoques. Uno de ellos es el llamado Teoría Racional Comprensiva y una derivación de éste llamada Teoría del Incrementalismo (Lindblom, 1980). Una de las críticas más fuertes hacia este tipo de enfoques la realizó Arrow (1963) en su exposición del Teorema de la Imposibilidad (Dunn, 1981). Es interesante apuntar que reflexiones como las de Harsanyi (:1993) asignan al principio de racionalidad el carácter de vinculo entre la eficacia y la eficiencia para lograr políticas que garanticen la equidad. Esta "omnipresencia" o ubicuidad del criterio de racionalidad, ratifica la conveniencia de abordario más como una condición que como un principio específico y distinguible de decisión. 
los formulados en los objetivos) en el bienestar de la población o de un sector de ella. La equidad se encuentra generalmente asociada con la justicia social, con los valores de igualdad y con una justa distribución y asignación de los recursos. Un equilibrio entre equidad y eficiencia está determinado por la concepción de sociedad que se tenga.

Lo que sigue, de ninguna manera pretende enunciar teoria alguna, sino más bien señalar una serie de suposiciones teórico-metodológicas sobre estos principios y su relación con el proceso de formulación de la política social. De estas líneas se desprende, entre otras cuestiones, que en un proceso de toma de decisiones que tenga que ver directamente con la formulación de una determinada política social, los actores responsables de su diseño, pueden privilegiar uno de estos criterios por sobre los otros.

\section{Eficiencla y eficacia: la justificación de los medios $y$ de los fines}

Son conceptos esenciales en la evaluación de una política y ayudan a establecer el grado de racionalidad en la asignación de los recursos que necesitan los proyectos y planes para ejecutar la política.

\subsection{Eficiencia}

Conocer sobre el desarrollo del concepto de eficiencia tiene un interés particular, ya que éste es frecuentemente utilizado, al mismo tiempo que se le da un peso preponderante, en el proceso de formulación de los objetivos de las políticas sociales así como también en su evaluación. En términos generales, la eficiencia se define, en el contexto de la formulación de las políticas públicas en general, como el diseño metodológico que privilegia la escogencia y selección de los medios sobre los fines.

Se ha argumentado que el paradigma dominante de análisis político encuentra sus raices en el principio del utilitarismo, tal y como fuera desarrollado desde el cálculo utilitario por Jeremy Bentham ${ }^{5}$, quien sostuvo que el objetivo prioritario de la política pública debia ser el logro de la mayor suma de felicidad para el conjunto de la sociedad. La eficiencia es entendida como un meta-valor que considera como dados los valores particulares que son perseguidos por los ciudadanos y por las instituciones públicas. El utilitarismo recomienda que cada vez que se tome una decisión se determinen las consecuencias e impactos asociados a ésta y se evalúe su eficiencia de acuerdo a su utilidad en los individuos afectados por esa política.

El corazón normativo del concepto de eficiencia según Bentham, (citado por Burms y Rosen, 1983) se deriva de un sistema político y social que diseña, construye y formula políticas públicas dirigidas a maximizar la satisfacción de las necesidades de todos los individuos que constituyen esa sociedad. Ello, a su vez, está suje-

Véase: J.H. Burms and F, Rosen (Eds.) 1983: The collected works of Jeremy Bentham, N.Y., Oxford University Press. 
to a ciertas limitaciones que tienen que ver, especificamente, con un incremento de las satisfacciones de las necesidades. El análisis llamado eficientista se apoya en los cálculos realizados por Bentham sobre la felicidad y el dolor, que son, según argumenta este autor, las dos fuerzas centrales de la condición humana.

Se le han señalado al menos tres tipos de objeciones o problemas a esta forma de construir y evaluar politicas públicas. La primera objeción señalada es la oposición que, frecuentemente, se presenta entre las necesidades que los individuos desean satisfacer y los límites significativos que señalan los recursos disponibles para satisfacerlas. La consecuencia de lo anterior se traduce en la capacidad que cada sociedad tiene para desarrollar los mecanismos necesarios con el objetivo de ajustar la brecha entre lo que quieren los individuos y los recursos disponibles para satisfacer sus necesidades.

La pregunta obligada que se le hace al análisis eficientista-utilitarista es cómo se deberían asignar los recursos dirigidos a satisfacer las necesidades entre los diferentes miembros de una sociedad. El problema aquí es la presencia, en los individuos, de utilidades dificilmente comparables entre sí. En una sociedad con recursos limitados, en donde los individuos compiten por ellos para satisfacer sus necesidades y mejorar su calidad de vida, podría ocurrir alguna de estas dos situaciones: a) que se presente un mejoramiento de la calidad de vida de un sector minoritario de la sociedad; b) que se presente un desmejoramiento de la calidad de vida de un sector mayoritario de la sociedad. Formalmente hablando, desde este punto de vista, lo que algunos individuos ganan en bienestar produce pérdidas de bienestar en muchos.

Siguiendo la argumentación utilitarista, el criterio de Eficiencia de Pareto ${ }^{6}$ (1919) llamado el Optimo de Pareto, nos indica que un sistema es eficiente cuando asigna los recursos disponibles de manera tal que una futura distribución de bienes no pueda incrementar las utilidades de unos individuos sin disminuir la utilidad de los otros. Una política pública dada es eficiente, según el sentido estricto de Pareto, cuando incrementa el bienestar de muchos sin disminuir el de otros. Dicho de otra forma, todos los individuos pueden ganar todas las utilidades posibles si al mismo tiempo crecen las utilidades de los otros. Para Pareto, si la utilidad de algunos individuos es producto o resultado de reducir las utilidades de los otros, el sistema no está operando con eficiencia. Una política dada es eficiente cuando aumenta el bienestar de al menos un individuo sin desmejorar el bienestar de otro. Es interesante anotar aquí, que este principio no contempla un punto igual de partida para los individuos ubicados en los diferentes sectores sociales en cuanto a la designación eficiente de los recursos. Suponemos que el proceso se inicia con una nueva redistribución de los recursos y por medio del criterio de eficiencia incorporado a una 
política se llega al punto óptimo que es el que incrementa el bienestar eliminando las diferencias entre los miembros de la sociedad.

El Optimo de Pareto impone severas restricciones a las acciones de los gobiernos. No es fácil beneficiar a una comunidad como un todo sin disminuir el bienestar de algún individuo (aquí se presenta el problema de la difícil comparación entre utilidades individuales antes señaladas). Una especificación y/o corrección al llamado Optimo de Pareto es el criterio desarrollado posteriormente por Kaldor-Hicks ${ }^{7}$ (1919). Este criterio señala que se permiten distribuciones que incrementen el bienestar neto de todos aquellos que ganan de la distribución general y que podrían compensar a aquellos que pierdan. Es decir, que lo que se redistribuye a los que pierden no ocasione pérdidas significativas a las utilidades de los que ganen a través de la distribución inicial. En este caso no sería necesario comparar entre las utilidades individuales ya que lo importante en este análisis es el valor que tiene la sociedad acerca de la redistribución de los bienes como un fin común y posible, siempre y cuando los bienes estén disponibles y sean redistribuidos de manera eficiente. El criterio especifica que es posible determinar de un modo justo el incremento de los que ganan y la compensación a los que pierden, de manera tal que la situación de bienestar de éstos últimos, debería ser mejor que la que pudiese resultar de no haber habido redistribución alguna.

El principio de eficiencia requiere de la compensación de la acción y es a través de ésta como se construye una red de beneficios a ser redistribuidos. La teoría no define los mecanismos a través de los cuales se haria efectivo este criterio, asimismo, obvia cómo debería ser realizada la compensación. A pesar de lo anterior, el argumento de análisis de eficiencia basado en el criterio de Kaldor-Hicks y su instrumentación, constituye el corazón del paradigma dominante del análisis valorativo de una política pública y por extensión de la política social. Con ello no queremos decir que éste sea el único criterio empleado en la formulación de una política, pero si el más utilizado para evaluar sus resultados e impactos.

La segunda objeción formulada a este principio viene dada por la dificultad de hacer comparaciones interpersonales a través de la noción de utilidad. Ante ello, Rawls (1979) propone sustituir utilidad por bienes básicos, es decir, por condiciones objetivas que hagan posible la obtención de la felicidad ${ }^{8}$. La tercera objeción tiene que ver con la naturaleza de las preferencias individuales que se constituyen en referencia obligada para la elec-

7 Existen diferencias entre el utilitarismo de Betham, el Optimo de Pareto y el Principio de Compensación de Kaldor-Hicks. J.R. Hicks 1939:"The foundations of welfare economics", The Economical Joumal, Vol.49, 느.196:696-712.

8 Para Rawis estos bienes entre otros serian: las libertades, posibilidades de acceso a posiciones sociales, los poderes y los ingresos. 
ción, aun cuando constituyan preferencias determinadas por el medio y otras formas de presión y/o control social.

En general, el análisis politico que privilegia el principio de la eficiencia es aplicable a cualquier formulación de política social, para cualquier sociedad en la cual los individuos buscan de una forma cooperativa las satisfacciones de sus necesidades. Esta búsqueda de satisfactores se ordena en forma de preferencias a ser obtenidas al menor costo posible; ello supone objetivos acordados y un conocimiento de las características del bienestar general deseado. La sociedad es entendida estrictamente como una agregación de individuos y el bienestar social está constituido por la agregación de los bienestares individuales. Una derivación de este enfoque es lo que se ha llamado la Teoría de la Elección Social ${ }^{9}$, que trata de superar las desventajas señaladas al utilitarismo de Pareto y explorar las ventajas a través de la imposibilidad de la comparación y la introducción en el análisis de la categoria de la indiferencia.

Sintetizando, la eficiencia es el principio que domina la formulación y evaluación de las políticas que tienen que llevarse a cabo con un número particularmente limitado de recursos. Tiene una influencia importante en la planificación y en este ámbito se le entendió como un proceso de diseño de soluciones a problemas cuyos costos debian ser lo menos onerosos posible en términos de recursos.

Las democracias liberales y las políticas públicas adoptadas por sus gobiernos priorizan, además de la eficiencia en la formulación y resultados de una política, la eficacia que poseen éstas en resolver los problemas que se presentan y su impacto en una población determinada. El principio de eficacia coloca el énfasis en la formulación de los fines $u$ objetivos de la política.

\subsection{Eficacia}

Cuando se habla de eficacia se está haciendo referencia a los aspectos que intervienen en la resolución de los problemas que se le presentan a la sociedad y cómo se alcanzan a través de una política determinada; el énfasis está puesto en la formulación y alcance de los fines de la política. Al respecto Cohen y Franco (1992) definen eficacia como el grado en que se alcanzan los objetivos y metas de la política en un período determinado, independientemente de los costos que ello implique.

Siguiendo la argumentación de Barragán al respecto señala que todo proceso de formulación de una política pública concieme, - además de estar relacionada con los instrumentos o medios para efectuar la distribución de los beneficios y las cargas sociales- también a los fines que están incluidos en la política que son los que la do$\tan$ de contenido y orientaciones precisas en cuanto a su instrumentación. Lo que subyace en la argumentación anterior, en palabras de Barragán (1992:68), es que formular una política no es otra cosa que "elaborar una decisión entre políticas alternativas, las que a su vez representan fines alternativos..." por lo cual no solamente es 
significativo discutir sobre los medios, sino que también se debe deliberar sobre los fines de la política en cuestión.

Si bien es cierto que la politica social tiene como fin general la construcción del bienestar colectivo mediante la redistribución de bienes escasos, no es menos cierto que esta acción no está libre de conflicto entre intereses que obligan a tomar en cuenta las diferencias y contradicciones que se presenten. Empero, en su carácter de principio normativo, la eficacia puede ser entendida "... como la exigencia de establecer precondiciones en los procesos decisorios que regulan la convivencia de una sociedad dada" (Alzuru, 1994:18).

En el sentido señalado, la construcción del bienestar colectivo a través de la política es extremadamente compleja. Taly como lo expone Barragán, para algunos el concepto de bienestar colectivo se relaciona con mecanismos desplegados por el Estado para aminorar las desigualdades que existen en una sociedad dada; otros lo asocian con el peligro que conlievan las restricciones relacionadas con la autonomía y los derechos individuales. En palabras de Barragán (1992:69) "...en la construcción de una función de bienestar colectivo hay que resolver la dura cuestión acerca de qué y cuánto de la propia función de bienestar individual podemos o estamos dispuestos a conceder a favor de la función de bienestar colectivo...". Se deduce entonces que de alguna manera el diseñador de una política social tendrá la responsabilidad de justificar la definición y selección de los fines que persigue dicha política.

El paradigma dominante en este campo deriva ciertas técnicas metodológicas que dirigen su atención hacia la construcción de políticas. Estas técnicas están fuertemente influenciadas por los principios de eficiencia y de eficacia y los criterios que de ellos se derivan presentando algunas bases normativas. La técnica que -tradicionalmente- goza de más popularidad entre los diseñadores de políticas guiados por estos dos principios es la llamada costo-beneficio: esencialmente tiene como objetivo el que la política genere más beneficios que costos sociales, partiendo del supuesto general de que los resultados de la política instrumentada sean óptimos. Otra de las técnicas frecuentemente utilizadas es la de costo-efectividad que tiene como uno de sus objetivos minimizar los costos y maximizar los resultados a la par de determinar el grado de eficacia y de eficiencia de distintas opciones de política o de diferentes políticas que persigan los mismos fines. Estas dos perspectivas tienen mucho que decir en cuanto a la selectividad de las políticas sociales ya que sugieren que el gasto sea dirigido hacia los más necesitados.

Otro modo de acometer la justificación de los fines y su correspondencia con los medios de una política, es incluyendo los principios de racionalidad y equidad en la discusión sobre las distintas opciones de política lo que implica evaluar las consecuencias e impactos de la decisión a tomar ${ }^{10}$.

\section{Equidad: la igualdad y la justicia}

Según el Diccionario de Filosofía de Abagnano (1974:420) el concepto clásico de equidad, tal y como fuera definido por Aristóteles reza que: "La naturaleza misma de la equidad es la rectificación de 
la ley cuando se muestra insuficiente por su carácter universal". Se podria interpretar que la equidad es un concepto con base jurídica de fundamento ético, equivalente al de justicia natural, igual $\mathrm{e}$ imparcial. Según esta misma fuente, para Aristóteles, siendo la equidad y la justicia la misma cosa, la equidad se muestra superior a lo justo formulado por la ley. Para nuestros efectos entenderemos la equidad en un sentido amplio que incluye o contiene de cierta forma los conceptos de justicia e igualdad. Aunque estos términos se usan frecuentemente de forma intercambiable, sin embargo, podemos hacer algunas diferencias entre ellos.

La igualdad nos dice acerca de que todos posean la misma cantidad de algún bien, las mismas proporciones. No tiene que ver con las variaciones en cuanto a las necesidades que pueda haber entre diferentes grupos sociales. Al principio de equidad se le añaden las dimensiones sociales de la justicia y la imparcialidad, lo que implica que va más allá de la idea de igualdad. Equidad sugiere que diferentes personas o grupos sociales deberian te- ner igual acceso a recursos o bienes de forma suficiente que satisfagan sus necesidades. Al mismo tiempo concierne con la igualdad de oportunidades para adquirir un bien y disminuir las diferencias entre los que tienen más ventajas y entre los que tienen menos ventajas al más bajo nivel posible. En condiciones de escasez de recursos, la equidad adquiere especial importancia sobre todo respecto de los bienes públicos 0 de carácter colectivo ${ }^{11}$.

Según algunos autores estudiosos de la evolución de este concepto (Gurrieri, 1990) equidad es sinónimo de justicia e igualdad; en esta orientación pretendemos analizar este principio ${ }^{12}$. La noción de equidad ha venido variando en el tiempo y como otros conceptos ha sido corregida, no solamente por la ideología dominante, sino también por el devenir sociopolítico particular de cada sociedad. Desde la definición clásica y simple de Aristóteles hasta la contemporánea y compleja noción de hoy día, la cual ya es mucho más compleja que el definir la equidad sólo como igualdad ante la ley. Sin embargo, queremos dejar claro que en este

10 Barragán anota que existen dos tipos de éticas: la ética de la convicción y la de la responsabilidad. En ambas se observan dificultades debido a la complejidad del proceso de toma de decisión. En la primera el decisor corre el riesgo de hacer coincidir su elección con los principios que le garanticen el menor costo posible y la maximización de las ganancias. La ética de la responsabilidad requiere que antes de seleccionar una política se tendrá que evaluar sus consecuencias. (1992) Otro modo de examinar esta problemática es a través de la ética voluntarista y la ética racionalista (Weber, 1972).

11 Elster (1994) analiza entre otros, el principio de igualdad, considerado como la línea de base para la distribución por diferentes filósofos y políticos, -la igualdad, en términos generales expresa lo que es justo-y los principios derivados de los niveles individuales de bienestar, entre los cuales se incluyen las necesidades, los incrementos en el bienestar y la eficiencia.

12 En palabras de Franco, la equidad no se debe confundir con la justicia y la igualdad. La justicia es un valor inalcanzable, la equidad es aquella parte de la justicia que es realizable en un momento histórico determinado: "...una sociedad equitativa no busca la igualdad de los resultados, pero pretende ir más allá de la igualdad ante la ley" (2000: 50-51). 
punto sólo nos interesa destacar y desarrollar las características esenciales de la equidad: la distribución, redistribución y asignación de recursos y su relación con la evaluación de las políticas sociales. Al mismo tiempo intentaremos articular las concepciones de racionalidad que se derivan de los enfoques de la equidad.

Con el objeto de mejorar nuestra comprensión sobre estos aspectos, nos apoyaremos en tres de las teorias politico-filosóficas contemporáneas, las cuales podrian ayudarnos a identificar patrones de decisión política que privilegien la equidad, ya que partimos del argumento de que el alcance de estas teorías es la regulación de la distribución y redistribución del bienestar colectivo. Estas tres teorías son: la teoría de la justicia de Rawls, la teoría libertaria de Nozick y las esferas de la justicia de Walzer ${ }^{13}$. Las tres teorias se abordarán de una forma breve y esquemática sobre los puntos arriba especificados, los demás problemas que presenten serán dejados de lado ${ }^{14}$. Con esta indagación nos interesa responder básicamente a dos cuestiones: 1) qué entienden por justicia estos autores y 2) a qué ámbito la refieren o pertenece. Complementariamente aludiremos a las articulaciones de estos enfoques con la racionalidad.

\subsection{Rawls: Justicia como equidad}

Partiendo de la tradición neocontractualista, Rawls concibe que "la idea de equidad es la idea fundamental en el concepto de justicia" (1986:18), desarrolla su teoria entendiendo la justicia como equidad y postula que ésta es una elección racional de los individuos, -que está implícita o latente en una sociedad democrática-, por lo que sustrae al Estado la exclusividad de la función compensadora ubicándola en el ámbito de la sociedad. Deslastrado de toda concepción moral, religiosa o filosófica, parte de una concepción substantiva de la justicia y elabora criterios que permiten apreciar grados de justicia a partir de las prácticas de las instituciones sociales que son los cuerpos por excelencia que la distribuyen hacia la sociedad.

El autor hace uso de una situación hipotética (la situación original) en la que los individuos eligen "principios" en un estado primordial de igualdad, sin saber su propia situación en los estados sociales que resulten de ella e ignorando incluso sus posiciones sociales lo que hace que actúen de forma neutral y desinteresadamente ("velo de la ignorancia"). En tal situación, el principio que sería aceptado por todos es el criterio de equidad, siendo el resultado de un acuerdo equitativo sin intereses creados. La sociedad la entiende como un sistema imparcial de cooperación social entre individuos libres e iguales que tienen un sentido de justicia y una concepción personal del bien común (a)truismo limitado). Por lo que la justicia

13 John Rawls escribe su trabajo Justicia como equidad, publicado en inglés en 1958 y en español, en 1986 y su Teoria de la justicia en 1979; Robert Nozick desarrolla este concepto en 1974 en su libro titulado Anarquía, Estado y Utopía y Michael Walzer en 1983.

14 Véanse entre otros a: Sandel (1998); Mouffe (1999); Elster $(1991,1994)$. 
política deberá desarrollar los principios adecuados para alcanzar la libertad e igualdad de sus miembros.

Esta concepción de justicia está conformada por dos principios fundamentales: 1) las libertades deben distribuirse por igual y 2) las desigualdades económicas y sociales deben ser reguladas a través de dos condiciones: a) el mayor beneficio debe ser para los menos aventajados $y$ b) todos deben tener igualdad de oportunidades para acceder a los bienes (bienes sociales primarios constituidos por derechos, oportunidades, ingreso, riqueza y bases sociales del respeto a si mismo). De lo que se trata es de asegurar que todos gocen de las mismas libertades y de las mismas posibilidades. El objeto de la justicia es la estructura básica de la sociedad, en palabras de Rawls "... el modo en que las instituciones sociales más importantes distribuyen los derechos y deberes fundamentales y determinan la división de las ventajas provenientes de la cooperación social" $(1979: 23)^{15}$ De este modo, la igualdad, base de la justicia, queda determinada por la libertad ${ }^{16}$.

Por tanto, a partir de esta concepción de la justicia se desprende que para estar en condiciones de evaluar la estructura básica de una sociedad debemos primero evaluar las prácticas y responsabilidades de sus principales instituciones en tanto cumplen funciones distributivas, por lo que esta teoría construye parámetros dirigidos a anular y neutralizar las distinciones arbitrarias y sus efectos asi como los grados de justicia producto de los arreglos políticos, sociales y económicos Rawls (1979).

En esta misma línea, para Rawls, el mercado es el lugar donde se tratan los problemas de la distribución. Distingue, en este espacio, el principio de la diferencia y el principio de la compensación. Este último tiene como finalidad asegurar la igualdad de oportunidades consecuencia de las desigualdades de nacimiento $y$ dotes naturales que necesariamente deben compensarse. EI principio de la diferencia impone restricciones a las ventajas provenientes de las oportunídades naturales y establece una base sobre la cual las distribuciones desiguales podrían ser consideradas justas, es el principio que ordena beneficiar a los miembros de la sociedad menos favorecidos. Un sistema justo es aquel que dado un sistema de libertades iguales para todos y una equitativa igualdad de oportunidades, ningún cambio en las expectativas de los más favorecidos puede desmejorar la situación de los menos favorecidos.

Como se desprende de las líneas anteriores, un sistema de instituciones justo debe incluir las condiciones expuestas en los principios mencionados. El primero es parte del segundo y a través del principio de la diferencia Rawis introduce

15 Como ejemplos de instituciones más importantes el autor entiende a la constitución politica y las principales disposiciones económicas y sociales, la protección jurídica de la libertad de pensamiento y de conciencia, la competencia mercantil, la propiedad privada de los medios de producción y la familia monogámica. Rawls (1979:23).

16 Entendida como igualdad de oportunidades y de beneficios. 
las consideraciones distributivas como criterios fundamentales para evaluar la politica social.

La teoria de la justicia de Rawls, ha sido difundida como no asistencialista, ni utilitarista. No se ocupa de promover un bienestar igualitario sino el que los individuos busquen su felicidad. Para ello necesitan de ciertos recursos o de bienes y éstos bienes deberian distribuirse según el principio de la diferencia. Lo que significa que se deben asignar recursos de manera tal que se maximice la cantidad de bienes primarios de los que tienen la menor cantidad de aquéllos. Pero como bien señala Victoria Camps (1990:24) "... la teoría de la justicia de Rawls se muestra insuficiente en aquellos países o sociedades en donde la justicia está 'bajo mínimos', donde la desigualdad es tan escandalosa, que lo más racional consiste en desconfiar de que ninguno de los principios lleguen a aplicarse nunca"17.

Cohen y Franco (1992) interpretan que de las nociones de equidad desarrolladas por Rawls se deriva que la política social deberá atender primero a los más necesitados. Entre el universalismo y la selectividad opta por la segunda, por cuanto el universalismo conduce a tratar de la misma manera casos diferentes. La equidad, según este enfoque, aconseja no aplicar la misma solución a situaciones distintas. Esta misma idea es reforzada por Mouffe (1999) cuando señala que Rawls insiste en un marco situacional para su teoría de la justicia.

La noción de racionalidad de este enfoque se deriva de la insistencia de Rawls en señalar que el objetivo de su teoría de la justicia es establecer un consenso factible, basado en principios compartidos de justicia que impliquen, necesariamente, la realización de valores políticos. Ello requerirá que los decisores manejen una concepción del bien común 'razonable' y 'racional' que permita el despliegue de un discurso coherente y tolerante dirigido a formular políticas en términos equitativos basados en la cooperación.

Aawls subordina lo racional a lo razonable, y lo razonable refiere a una concepción de los términos de la cooperación social a través de reglas neutrales y acordadas, pero con carácter moral, ya que es imprescindible poner límites a la prosecución ilimitada del interés individual. Por último, tal y como lo señala Mouffe (1999:76) Rawls ha "usado un modo de razonamiento especiffco del discurso moral cuyo efecto, al aplicarfo al campo de la política, es reducir este último a un proceso racional de negociación entre intereses privados con las limitaciones impuestas por la moral. De esta manera, simplemente desaparecen, conflictos, antagonismos, relaciones de poder, formas de subordinación y represión y nos encontramos ante una visión típicamente liberal de una pluralidad de intereses que se pueden regular sin necesidad de una instancia superior de decisión política en que se evacue la cuestión de la soberania".

\subsection{Nozick: Teoria libertaria de la justicia}

Nozick, al contrario de Rawls, no parte de un concepto substantivo de 
justicia, sino que desarrolla su argumentación a partir de la concepción libertaria de las organizaciones de la sociedad. Construye su teoria de acuerdo con dos argumentos básicos: uno negativo, que incluye una crítica contundente a las teorías de justicia que, como la de Rawis, adoptan patrones o criterios para juzgar una mala distribución, y por tanto presumen cuál debería ser el papel de las agencias distributivas centralizadas, y el argumento positivo, construido en torno a la tentativa de mostrar que un Estado mínimo justificado a partir de la anarquia (el estado de naturaleza), no viola los derechos individuales, mientras que cualquier Estado más extenso que el mínimo, viola los derechos de las personas (Nozick 1988).

El argumento positivo tiene su base en la visión de que la redistribución a través del sistema fiscal es inmoral y se dispone a mostrar cómo un Estado mínimo es altamente moral y no va en contra de los intereses de los individuos ya que la mano invisible, conformada por las agencias protectoras privadas, tiene como objetivo proteger a los individuos impidiendo que unos usen procedimientos no confiables en contra de los otros en cuanto al proceso de distribución de recursos y bienes se refiere. A esta primera etapa la denomina el Estado ultramínimo, el cual está encargado de proteger y velar por aquellos que están al cuidado de las agencias protectoras, ya que pagan por ese servicio. Posteriormente, después de un proceso de lucha por el poder daría lugar a una agencia protectora dominante que sería el Estado mínimo, el cual tendría el monopolio de la provisión de los servicios de protección que serian de ca- rácter universal, lo que configura la función redistributiva del Estado mínimo. Sólo un Estado como este puede ofrecer una protección efectiva a todos los miembros de una sociedad y por tanto ello sería la garantía para la que distribución instrumentada sea justa.

Para Nozick, la idea de justicia no implica la utilización de mecanismos que tengan criterios de distribución. De esa forma no admite distribuciones desde agencias centralizadas, ni redistribuciones basadas en algún criterio específico. Para este autor, ninguna persona o grupo tiene derecho de controlar recursos; 10 que cada persona recibe, lo recibe de otros a cambio de algo. Una distribución dada, por tanto, es producto de muchas decisiones individuales que diferentes individuos tienen derecho a tomar. Sobre este punto elabora un argumento de tipo moral para justificar la protección a aquellos que no pagan, de esta forma lo que bien podría ser interpretado como redistribución él lo entiende como compensación.

Nozick (1988: 154-ss) identifica dos principios de justicia distributiva: históricos y de resultado final. Los primeros se refieren a los origenes de una situación dada y sostiene que "... las circunstancias o acciones pasadas de las personas pueden producir derechos diferentes o merecimientos diferentes sobre las cosas" Nozick (1988:158). Los segundos conciernen a las variaciones de la distribución en el tiempo. Ahora bien, para darle contenido a la concepción de justicia que se deriva de los dos principios mencionados, Nozick formula dos principios útiles para especificar la naturaleza de los derechos fundamentales: 
1) El principio de adquisición original de pertenencia, sostiene que cada uno puede apropiarse legitimamente de algo que no haya pertenecido anteriormente a nadie, siempre que el bienestar de ningún otro individuo se encuentre disminuido por ese hecho, y 2) El principio de transferencia, plantea que cada uno puede convertirse en el propietario legitimo de una cosa adquiriéndola por medio de una transacción voluntaria con la persona que era antes su propietaria legítima. Nozick (1988: 169).

Al contrario de Rawls, Nozick defiende la idea de un Estado que se limite a garantizar el cumplimiento de la ley $y$, mantener el orden, sustrayéndole la función distributiva. La racionalidad podrá desarrollarse a partir de un sujeto moral que sea capaz de distinguir lo bueno de lo malo, lo justo de lo injusto y de ningún modo podrá haber una prioridad del derecho sobre el bien. La sociedad es entendida como un agregado de individuos que decidieron asociarse (situación originaria) contratando a un Estado mínimo cuya tarea principal es proteger los derechos de los individuos.

En este sentido, Nozick trata de demostrar en su teoria que hay un conjunto de razones (racionales) y que son de carácter obligatorio para todos los individuos que persiguen su propio interés bien entendido. La explicación sobre lo que es racionalidad para este autor, está dada en la medida en que los individuos participan en un mercado $y$ no lo hacen únicamente por motivos egoistas, sino también sobre la base de contratos particulares que podrían Ilegar a convertirse en un contrato social amplio que no pase por el Estado, sino más bien como parte de la celebración simultánea de contratos entre una pluralidad de individuos (Klient, 1979).

\subsection{Walzer: Las esferas de la justicia. Una defensa del pluralismo y de la igualdad}

Parafraseando a Gordon (1995: 183) Walzer pretende desarrollar la posibilidad de justicia social a través de la instrumentación de criterios diferenciados y autónomos que pertenecen a los diversos ámbitos de la sociedad, argumentando que no es deseable la utilización de un criterio único de justicia para toda una sociedad concluyendo que existen diversas esferas de justicia. Elabora una concepción de justicia de carácter plural y a partir de una teoria de la igualdad compleja en la que la sociedad es vista como un vasto sistema de distribución que proporciona todo tipo de bienes materiales y no materiales, económicos y no económicos, como la salud, la educación, la seguridad, la identidad nacional, la ciudadanía, e incluso la pertenencia, el poder y el honor, entre otros. Al mismo tiempo, se generan mecanismos de diferenciación en donde hay agentes (mercado, Estado, familia, burocracia) que distribuyen bienes y recursos a través de ciertos criterios (mérito, necesidades, lealtad, política). Waizer parte de la idea de que los bienes sociales tienen significados distintos, y los criterios y procedimientos distributivos son intrínsecos respecto al bien social a ser distribuido. No contempla este autor normas únicas de distribución de los bienes sociales sino que más bien cada esfera de justicia genera sus normas particula- 
res de distribución de los bienes tomando en consideración su naturaleza (Walzer 1983).

Plantea diferencias respecto de la aplicación de reglas formales de distribución, como las que postula Rawls. Con ello, no acepta que el mercado con su lógica de regulación y distribución económica, sea el que garantice la distribución de los bienes colectivos en una sociedad. EI mercado, para Walzer, es una esfera sin fronteras, el dinero incide en las relaciones y las hace expansivas. El mercado tiende a invadir otras esferas y dominar los poderes distributivos.

En su línea de argumentación, que consiste en evitar toda pretensión de construir un enfoque de justicia distributiva basado en un criterio único de distribución, Walzer (1993:34,37y 38) desarrolla sin embargo tres criterios que aparentemente cumplen con los requisitos de principios abiertos pero ninguno de ellos cumple con toda la gama de distribuciones; ellos son: intercambio libre, merecimiento y necesidad. El primero es un criterio abierto y no garantiza ningún resultado distributivo en particular. El autor señala que al menos en teoria, este principio "...crea un mercado en que todos los bienes son convertibles en todos los otros bienes a través del medio neutral del dinero". Cada intercambio realizado revela un significado social y deja las distribuciones en manos de los individuos. El segundo, el merecimiento, al igual que el criterio anterior es abierto y diverso y exige juicios difíciles; aún así, este principio, sólo en condiciones muy especiales, produce distribuciones específicas. El tercer principio genera una esfera distributiva particular dentro de la cual la necesidad es el principio distributivo apropiado; en palabras del autor: "En una sociedad pobre, una gran proporción de riqueza social seria llevada hasta esta esfera." Pero debido a la variedad de bienes, otros criterios distributivos deben ser utilizados paralelamente al de la necesidad.

Habiendo establecido que las diferentes esferas de la sociedad obedecen a diferentes principios distributivos, que la instrumentación de un principio no debe traspasar las fronteras de una esfera determinada y que ningún bien social debería de servir como medio de dominación, pasa a examinar las diferentes esferas de justicia a partir de los bienes a ser distribuidos: la seguridad, el bienestar, el dinero, los cargos, la educación, el tiempo libre y el poder político. La idea de justicia distributiva para Walzer presupone un mundo con demarcaciones (esferas) dentro del cual se dan las distribuciones. A este lugar (comunidad política) lo define conformado por un grupo de hombres y mujeres que intercambian y comparten los bienes sociales, se distribuyen el poder entre sí y evitan, cuando pueden, compartirlo con alguien más. El bien primario que se distribuyen en primera instancia es el de la pertenencia, y será ésta la que estructurará las opciones distributivas. Inmediatamente están los bienes que se producen en las esferas de la seguridad (previsión comunitaria) y del bienestar que son los que enseñan el valor de la pertenencia y a su vez ello implica una relación con el criterio de necesidad; $y$ con base en las especificaciones que de éste se derivan, Walzer construye un argumento calíficado de igualitario (Cheibub, 1997), esto es que, una distribución real será limitada por los recursos 
disponibles, éstos a su vez son un producto del pasado y presente y no de algún excedente de riqueza; las necesidades socialmente reconocidas constituyen la primera demanda sobre el producto.

Walzer, a diferencia de Rawls y Nozick, presenta una teoría pluralista de la justicia social cuya meta es la realización de la 'igualdad compleja' a través de la cual trata de conciliar la legitimidad de las decisiones políticas con la eficiencia de las mismas. Por lo que podríamos derivar que la racionalidad para este autor es una construcción social. En las Esferas de la Justicia desarrolla principalmente una teoría de carácter normativo. Los bienes son distribuidos mediante principios diferentes, la naturaleza de los bienes exige cierto principio de distribución y una justa distribución depende del significado que dicho bien tenga en una sociedad determinada. La racionalidad se construye a partir de las microdistribuciones que se suceden en las distintas esferas de la sociedad (Elster, 1994).

\section{Comentarios finales}

A modo de síntesis, lo que anteriormente se presentó constituye un conjunto de consideraciones sobre algunas formulaciones teóricas a partir de las concepciones de eficiencia, eficacia, equidad y racionalidad. Estos principios fueron expuestos, no tanto como prácticas que despliegan un sistema normativo a seguir a partir de valores, sino más bien como condicionantes de los sujetos (decisores) que tienen la responsabilidad de construir espacios públicos a través del diseño de políticas distributivas y redistributivas diri- gidas hacia la construcción del bienestar colectivo.

En cuanto a la relación entre eficiencia, racionalidad y equidad en una política social, Cohen y Franco (1992) hablan sobre diferentes opciones: entre la racionalidad y la supuesta opción entre equidad y eficiencia. Esta supuesta opción la definen a partir de tres planos diferentes: el primero de ellos corresponde a la perspectiva general, el segundo a los fines de la política social y el tercero y último hace referencia a la instrumentación de la política y su éxito. En el primer plano, el más abstracto, el dilema entre equidad y eficiencia no deberia de existir ya que la equidad se basa en valores y la eficiencia está definida como un instrumento para alcanzar los fines que la sociedad ha establecido y que parte de ellos corresponde cumplir a la política social.

De este planteamiento se deriva que la eficiencia implica la aceptación de los valores, por lo que la equidad está relacionada con dos cuestiones básicas: la redistribución, y la segunda, la distribución. Cohen y Franco (1992:24), analizan la relación de estos principios en la política social distinguiendo tres tipos de política: asistenciales, inversión en recursos humanos y promocionales. Las primeras, denominadas asistenciales, estarian, según estos autores, regidas por el principio de equidad, ya que su fin es el de elevar el nivel de bienestar de un sector de la población, que es en definitiva la parte de la sociedad que necesita de la política. En cuanto a las segundas, ellas responden a las demandas de la política social la cual requiere de una población que asegure la continuidad y desarrollo de la sociedad 
como tal. Los autores señalan que es el principio de la equidad el que rige estas políticas, pero sólo cuando se refieren al corto plazo y a un sector definido, mientras que el principio de la eficiencia predomina cuando la política se plantea para el mediano y largo plazo. En cuanto a las terceras, las intervenciones con carácter promocional, el principio que las guía es la eficiencia, el fin de las mismas es el que las caracteriza, tanto en el corto como en el mediano y largo plazo.

La perspectiva teórica anterior nos permite arriesgarnos a concluir que la política social tiende a ser formulada siguiendo una combinación de estos tres principios bajo dos premisas diferentes entre si, más no contradictorias: los principios normativos que tienden a definir los objetivos a seguiry otros aspectos de tipo más bien fáctico que señalan la forma de alcanzar los objetivos establecidos y nos dicen si es factible o no alcanzarlos. Ambas dimensiones son básicas para el diseño de una política social. Los hechos por si solos no dicen cómo resolver e intervenir en la situación. Su participación llega hasta la discusión sobre los medios y nos dicen muy poco sobre los fines. Por el contrario, los principios normativos discuten, no solamente sobre los medios, sino también sobre los fines.

En la evaluación de una política social concurren los medios y los fines. Una evaluación de tipo económico hará énfasis en la eficiencia del impacto de dicha política y no incluirá elementos distributivos en su análisis. Una evaluación de tipo social propiamente dicha incorporará principios de tipo distributivo en su evaluación; aquí se estaría intentan- do integrar en el análisis la eficiencia con la equidad.

Si partimos de una consideración en la cual el Estado tiene el deber de proteger a los individuos frente a cierto riesgos, que debería garantizar un mínimo de existencia, de satisfacción de las necesidades básicas, otorgar oportunidades para la satisfacción de las necesidades y en situaciones de escasez tendría que ser capaz de jerarquizar las necesidades y darles resolución en el ámbito colectivo, ¿cómo debería hacerlo? Ello constituye un problema complejo y a la vez está intrínsecamente relacionado con la condición económica de la sociedad. Una forma de responder a esta pregunta sería a través del desarrollo e instrumentación de políticas sociales. La racionalidad podría proporcionar una orientación en el sentido de que no es posible aceptar exigencias normativas que sean inconciliables con los juicios racionales y morales que dicta una sociedad.

Cuando evaluamos el proceso de toma de decisión de una política social desde la dimensión económica, estamos diciendo que la política tiene como principio rector a la eficiencia. El papel del Estado estaría limitado a controlar e intervenir en la asignación de los recursos, sería un guardián que vela por la seguridad y cumplimiento de la ley. Sus funciones sociales serían aquellas en las cuales no existe competencia alguna con el mercado o con otros ámbitos de la sociedad. La sociedad acorde con este modelo de Estado, sería definida de forma atomista, como una suma de individuos. Vista así, la estrategia de la descentralización, por tomar un ejemplo, no tiene cabida, ya que 
es definida como una forma de debilitar al poder central que se considera excesivo.

Una de esas 'constelaciones de afinidades' es la que se basa en la preferencia por el colectivo (descentralización, participación, servicios universales administrados por el Estado), otra constelación pretende la superación de la autonomía entre los diferentes principios, con una base racional destinada a solventar la oposición entre estos (fortalecimiento de la sociedad civil, reconocimientos de cuerpos intermedios de diferentes tipos).

Las opciones, en cuanto a principios normativos se refiere, conforman "constelaciones de afinidades electivas" en el sentido de que hay una probabilidad relativamente elevada de que cuando se decide la adopción de una determinada politica social se estará privilegiando uno de los principios descritos anteriormente, pero también podría ser que se presentara una combinación o coincidencia, así como también conflictos y contradicciones entre los principios elegidos. Es altamente probable que esas 'constelaciones' estén estrechamente vinculadas con las grandes líneas de corrientes de pensamiento vigentes para cada período sociopolítico.

\section{Bibliografia citada}

Aguilar Villanueva, L. (1988) "En torno al concepto de racionalidad de Max Weber", en: Olivé, León (comp.) Racionalidad, Siglo XXI edts. México, pp.76-98.

Alzuru, Alexis (1994) Racionalidad política y legitimidad del Estado, Monte Avila Editores/CELARG, Caracas.

Anderson, Charles (1979). "The place of Principles in Policy Analysis". The Ameri- can Political Review, vol, 73(3):711. 723.

Arrow, Kenneth (1963). Social choice and individual values, $N, Y$, John Wiley.

Barragan, Julia (1992) "La estructura de justificación de las políticas públicas en un marco democrático", en: Rey, J. C; Barragán, J, Y Hausmann, R.; América Latina. Alternativas para la democracia. Monte Avila Editores, pp. $51-86$.

Burms, J.H.; Rosen, F. (Edts) (1983). The collected works of Jeremy Bentham, N.Y., Oxford University Press.

Camps, Victoria (1990). Introducción al libro Sobre las libertades (de Rawis), Piadós/ICE-UAB, Buenos Aires, pp.9-25.

Cheibub, Argelina (1997). "Principios de justicia e a avaliacao de políticas", Lua Nova, 29:17-104.

Cohen, E; Franco, R. (1992). Evaluación de proyectos sociales, Siglo $X X 1$ Editores, México.

Dunn, William (1981). Public Policy Analysis. An introduction. Prentice-Hall, Inc. Englewood Cliffs, N.J.

Dunn, Willam (1988). "Methods of the second type: coping with the wilderness of conventional policy analysis", Policy Studies Review, vol 17,4:720-737.

Durkheim, E. (1973). Las reglas del método sociológico, Schapire Ed., Argentina.

Elster, Jon (1988). "La posibilidad de una política racional", en, Olivé, León (comp.) Racionalidad, Siglo $X X \mid$ edts. México, pp.132-176.

Elster, Jon (1991) . Juicios Salomónicos, Gedisa, España

Elster, Jon (1994). Justicia Local, Gedisa, España.

Franco, R. (2000). "Globalización, democracia y equidad. ¿Qué pasa hoy en América Latina?" Contribuciones, 3:49-73. 
Gauthier, David (1986). Morals by Agreement, Oxford University Press, USA.

Gordon, Sara (1995). "Equidad y justicia social", Revista Mexicana de Sociología, 2:175-184.

Gurrieri, Adolfo (1990). "La equidad: aspectos conceptuales y tareas futuras", CEPAL, Santiago de Chile, mimeo.

Harsanyi, J.C. (1993). Etica y politica en la decisión pública, Angria Ediciones, Caracas.

Hicks, J.R. (1939). "The foundations of welfare economics", The Economical Journal, Vol 49:196:696-712.

Klient, Hartmut (1979). Filosofía del Estado y criterios de legitimidad, Edt Alfa, Barcelona/Caracas.

Lindblon, C. (1980). The policy-maiking process, Englewood Cliffs, N.J. Prentice-Hall, Inc.

Mouffe, Chantal. (1999). El retorno de lo político. Comunidad, ciudadenía, pluralismo, democracia radical, Paidós, Buenos Aires.

Nozick, Robert (1988). Anarquía, Estado y Utopia. FCE, México.
Olive, León (Comp.) (1988). Racionalidad. Ensayos sobre la racionalidad en ética y política, ciencia y tecnologia. Edit. Siglo XXI. México.

Pareto, W. (1919). Manual de economia politica, Milan, Piccola Biblioteca Cientifica.

Perez Schael, Maria Sol (1995). "Democracia, ética y libertad. Hipótesis para analjzar el caso venezolano", Revista Venezolana de Economía y Ciencias Sociales, 2-3:26-48.

Rawls, John (1986). Justicia como equidad. Materiales para una teoría de la jus. ticia, Tecnos, Madrid.

Rawis, John (1979). Teoría de la Justicia, FCE, México.

Sandel, Michael (1998). Liberalism ahd the limits of justice, Cambridge University Press, United Kingdom.

Sen, A. K. (1970). Collective choice and social welfare, San Francisco University Press, U.S.A.

Walzer, Michael (1993). Las esferas de la justicia. Una defensa del pluralismo y la igualdad, F.C.E, México. 